

\section{Processo Formativo-Reflexivo Para o Uso Racional de Medicamentos nos Assentamentos do Município de Pontão/Rs: O Relato de Uma Experiência à Luz dos Ensinamentos Freireanos}

Formative-Reflective Process for the rational use of medicines in the Settlements in the City of Pontoon/RS: a report of an experience the Light of Freire's Teachings

\section{Resumo}

Este relato de experiência descreve a vivência de um grupo de pessoas formado por acadêmicos do curso de Medicina da UFFS/Campus Passo Fundo, docentes, colaboradora técnico-administrativa e preceptor do município de Pontão-RS, em um projeto de extensão com foco no uso racional de medicamentos para uma população de assentados desse município. Para os trabalhos foi utilizada uma forma específica de metodologia com base nos ensinamentos freireanos, o que fundamentou teoricamente a atividade. As atividades foram desenvolvidas ao longo de seis encontros com a população selecionada, em um espaço comunitário dentro do assentamento da antiga Fazenda Anonni, onde foram trabalhados os temas relacionados ao uso racional dos medicamentos. A vivência evidenciou a necessidade de uma abordagem continuada pelos profissionais de saúde acerca da importância do uso racional de medicamentos e dos perigos da automedicação. Durante o desenvolvimento das atividades do projeto foram sendo adaptadas à dinâmica e à linguagem utilizada para que se obtivesse o máximo de participação dos assentados. Concluímos que o desenvolvimento desse projeto de extensão possibilitou uma efetiva comunicação com a comunidade dos assentados, permitindo uma troca de saberes em que conseguimos conhecer um pouco mais sobre a realidade de vida e suas percepções sobre o uso dos medicamentos, bem como, construir conjuntamente conhecimento acerca dos temas propostos. Nesse mesmo sentido foi constada a importância da utilização do processo formativo reflexivo, defendido por Freire, para a formação de uma consciência sobre o uso racional dos medicamentos.

Palavras-chave: Processo formativo-reflexivo. Uso racional de medicamentos. Assentados. Extensão universitária.

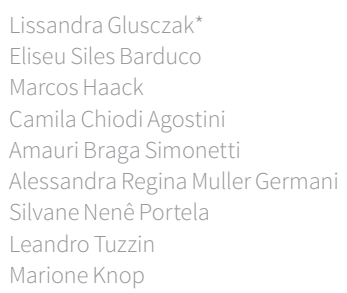


REVISTA GUARÁ - Dezembro 2018. ANO VI - NX

Fotografia 1 Manifestação Guarani contra Itaipu.

Fonte: Cimi (1991)
Abstract

This experience report describes the experience of a group of five people consisting of students from the medical school of UFFS / Campus Passo Fundo, teachers, a technical-administrative collaborator and a governess in the city of Pontão-RS, in an extension project with focus on the rational use of medicines to a population of settlers of this county. For the work we used a specific form of methodology based on Freire's teachings, which theoretically grounded the activity. The activities were developed over six meetings with the population selected which were accomplished in community space within the settlement of the old farm Anonni, where topics related to the rational use of medicines were being worked out. The experience highlighted the need for continued approach by health professionals about the importance of rational use of drugs and the dangers of self-medication. During the development of the project activities were being adapted to dynamic and the language used so that they obtain maximum participation. We conclude that the development of this university extension project enabled effective communication with the community of settlers, allowing an exchange of knowledge where we know a little more about the reality of life and their perceptions about the use of medicines, and build together knowledge the proposed themes. In the same vein revealed as being the importance of using the reflective learning process defended by Freire for the formation of awareness on the rational use of medicines.

Keywords: Formative-reflective process. Rational use of medicines. Settlers. University Extension. 


\section{INTRODUÇÃO}

Os medicamentos desempenham um papel estratégico na sociedade moderna, pois se converteram em elemento chave para minimizar o sofrimento humano. São recursos que produzem cura, evitam doenças, prolongam a vida, retardam o aparecimento de complicações de doenças, facilitando o convívio entre os indivíduos e suas doenças (LEITE, et al, 2008 apud PEPE, 2000). Se por um lado os medicamentos, em suas mais diferentes classes, são potentes à recuperação e manutenção da saúde, por outro lado seu uso indiscriminado tem gerado um cenário que se torna mais preocupante a cada dia.

Fatores como a propaganda massiva, o acesso facilitado à compra e a disponibilização, nem sempre confiável, de informações acerca das condições de saúde e o seu tratamento têm proporcionado um consumo descontrolado de muitos fármacos. Esses fámacos, mesmo com um bom perfil de segurança, dentro dessa dinâmica de uso tornam-se um risco potencial à saúde da população. Por outro lado, mesmo quando os medicamentos são adequadamente prescritos, muitas vezes a falta de orientações por parte dos profissionais de saúde ou a não compreensão das informações recebidas pelos usuários, pode facilitar a presença dos efeitos adversos e até sérias consequências à saúde dos usuários (OENNING, 2011).

Preocupados com essas questões, é que um grupo de professores desenvolveu o projeto de extensão "Uso Racional de Medicamentos: processo formativo nos assentamentos do município de Pontão-RS", realizado entre os anos de 2015 e 2016, com acadêmicos, docentes e técnicos administrativos em educação, vinculados à Universidade Federal da Fronteira Sul - UFFS - Campus Passo Fundo/RS junto ao assentamento de agricultores do Município de Pontão/RS.

Para esse projeto objetivou-se informar a comunidade da região sobre os cuidados com o uso racional de algumas classes medicamentosas e, além disso, possibilitou a troca de informações, entre estudos científicos do meio acadêmico com os conhecimentos empíricos do assentamento, fortalecendo o compromisso social da universidade na interação ensino-serviço-comunidade.

Dentro desse contexto o projeto buscou por uma alternativa metodológica, possibilitando o desenvolvimento de um processo formativo-reflexivo acerca do uso racional de medicamentos nas áreas de assentamentos no município de Pontão/RS, prevenindo a população sobre os riscos do uso inadequado de medicamentos. Tal processo pressupõe uma formação coletiva dos saberes, sendo que os encontros foram guiados nos sentido de coligar o conhecimento "popular" com o conhecimento científico, a fim de conjugar conhecimento crítico do sujeito, no que se refere ao uso de medicamentos.

\section{METODOLOGIA}

As atividades expostas neste trabalho basearam-se na vivência acadêmica do grupo composto por dois acadêmicos do curso de Medicina, dois docentes e uma técnica administrativa, todos oriundos da Universidade Federal da Fronteira Sul (UFFS) e uma preceptora, a qual é enfermeira da unidade básica de saúde do mu- 
nicípio de Pontão. Os acadêmicos e a técnica administrativa ficaram responsáveis por desenvolver material teórico audiovisual de apoio e atuar no papel de facilitadores na execução das atividades. Os docentes além de supervisionar a produção do material a ser utilizado e participarem também como facilitadores nas atividades com os participantes, ficaram responsáveis pela viabilização logística das atividades como transporte, ajustes nas datas e horários dos encontros, mantendo para isso contato contínuo com a preceptora do município, que atuou como contato direto com os moradores do assentamento que participaram do processo formativo.

Para a realização desses encontros foi necessária, também, a colaboração da equipe do Centro de Referência de Assistência Social (CRAS) e dos assistentes de saúde do assentamento do município de Pontão-RS. As orientações sobre ou uso de medicamentos foram ministradas por professores orientadores e acadêmicos da UFFS, as quais tiveram, em sua maioria, atividades e rodas de conversas que estimularam a participação de todos os presentes nos encontros.

Os objetivos compreenderam a realização de encontros programados com o público-alvo composto por sessenta moradores no total, oriundos de duas áreas dos assentados do município. Por meio de uma dinâmica ativa objetivou-se a abordagem de seis grandes temas norteadores para o processo formativo: apresentação do curso e automedicação, interações medicamentosas, uso racional de antidepressivos, uso racional de anti-hipertensivos, uso racional de anti-inflamatórios não-esteroidais e por fim o tema sobre plantas medicinais.

Isso possibilitou trocar conhecimentos com a comunidade da região sobre a importância e o cuidado com o uso racional das diversas classes medicamentosas. Essa comunidade inclui os assentados, o grupo da terceira idade, agentes de saúde e alunos do ensino fundamental. Esses encontros também foram importantes para realizar a troca de informações, de estudos científicos e/ou baseado em conhecimentos da comunidade adquiridos, de forma empírica, naquela região.

As atividades foram realizadas no assentamento da Fazenda Annoni, interior do Município de Pontão - RS, dentro do ginásio de esportes. Esse assentamento que é oriundo do Movimento dos Trabalhadores Rurais Sem Terra (MST) se tornou conhecido nacionalmente com a ocupação da fazenda por 2500 famílias em outubro de 1985. Após anos de discussões na Justiça e de embates dentro da fazenda, 430 famílias se estabeleceram definitivamente, aumentando a população da região e permitindo a emancipação de Pontão. Sendo assim, atualmente a cidade é predominantemente envolvida com a agricultura, e isso, sem dúvida, colaborou para o melhor resultado das atividades desenvolvidas naquela região.

Os encontros foram voltados para a construção coletiva de conhecimentos acerca do uso racional de medicamentos através de processo formativo-reflexivo visando através deste agregar saberes ao conhecimento prévio dos participantes acerca dos temas propostos, bem como a partir disso torná-los multiplicadores desses conhecimentos dentro da comunidade onde estão inseridos. 


\section{DA FUNDAMENTAÇÃO TEÓRICA}

Segundo a Organização Mundial da Saúde (OMS, 1985) o "uso racional de medicamentos implica em que o paciente receba o medicamento apropriado às suas necessidades clínicas, por um período adequado de tempo e com o menor custo possível para si e a comunidade" (MSH, 1997; CHAVES et al, 2005). A promoção do uso racional de medicamentos está inserida nas diretrizes da Política Nacional de Medicamentos do Brasil (BRASIL, 1998) e está envolvida no comprometimento de vários atores dentro e fora do setor saúde.

No Brasil, o uso incorreto de medicamentos geralmente deve-se ao uso indiscriminado de antibióticos, prescrição não orientada por diretrizes, automedicação indevida e inapropriado armamento terapêutico disponibilizado no comércio pelas indústrias farmacêuticas (WANNMACHER, L., 2012). Pesquisas comprovam que 35\% dos medicamentos adquiridos no Brasil ocorrem através da automedicação (BARROS, et al., 1995).

A preocupação com o crescente consumo de medicamentos se deve ao fato de que a grande maioria causa reações adversas (RAM) ou efeitos colaterais, muitas vezes mais graves do que a própria doença original. Segundo alguns dados acerca do uso irracional de medicamentos no Brasil, aproximadamente um terço das internações ocorridas no país tem como origem o uso incorreto de medicamentos (AQUINO, 2007).

Nesse projeto de extensão, foi utilizado como base teórica e condutora metodológica o Método Paulo Freire (1979, 1980, 1999 e 2001), no qual a construção dos saberes é realizada de forma coletiva. Freire (2011) propõe uma nova concepção pedagógica, em que a educação não é apenas a transmissão de conteúdos por parte do educador e sim uma relação de diálogo, no qual aquele que educa está aprendendo também, mediatizado pelo mundo. Nesta perspectiva, Gadotti (2000) elucida que o diálogo consiste em uma relação horizontal e não vertical entre as pessoas implicadas, entre as pessoas em relação. No seu pensamento, a relação homem-homem, homem-mulher, homem-mulher-mundo são indissociáveis e é por isso que os humanos se educam juntos na transformação do mundo.

O mesmo autor refere que outro tema relevante da obra de Paulo Freire é a conscientização que não é apenas tomar conhecimento da realidade através da análise crítica, mas sim o desvelamento das razões de ser dessa situação, para constituir-se em ação transformadora dessa realidade. Nesse sentido:

A curiosidade como inquietação indagadora, como inclinação ao desvelamento de algo, como pergunta verbalizada ou não, como procura de esclarecimento, como sinal de atenção que sugere alerta faz parte integrante do fenômeno vital. Não haveria criatividade sem a curiosidade que nos move e que nos põe pacientemente impacientes diante do mundo que não fizemos, acrescentando a ele algo que fizemos (FREIRE, 2001, p. 53). 
Mas o ponto central da sua concepção educativa é a libertação, sendo reconhecida como fim da educação, quer dizer, a finalidade da educação será libertar-se da realidade opressiva e da injustiça visando à transformação radical da realidade, para melhorá-la, torná-la mais humana e para permitir que homens e mulheres sejam reconhecidos como sujeitos da sua história e não, como objetos. Freire pensou em um método de educação baseado no diálogo que consiste em uma relação horizontal e não vertical entre as pessoas, com vistas a transformar a realidade refletida (FREIRE, 2011).

Nesse sentido, Freire nos mostra a importância da construção coletiva do conhecimento, a fim de construirmos uma concepção crítica de como o mesmo vem ou foi sendo desenvolvido a fim de aprimorar as próximas práticas advindas dele. Tal sistemática é crucial quando se trata de uso racional de medicamentos, a fim de que o conhecimento angariado faça entender a real necessidade e o momento certo para a aplicação do fármaco. O processo formativo-reflexivo também é crucial na decisão e na libertação daquele que o observa, já que o mesmo não executará mais atos apenas pelo simples fato de que alguém ou de que todos fazem dessa forma, mas sim após ponderar a situação, principalmente com base nos conhecimentos que detêm sobre o assunto.

Assim, é nesse arcabouço teórico que o trabalho foi desenvolvido, sendo que a metodologia pode ser aplicada plenamente na prática, restando em benefícios mútuos aos participantes do projeto, como será visto no relato da experiência a seguir.

\section{O RELATO DA EXPERIÊNCIA: OS RESULTADOS}

O projeto intitulado "Uso Racional de Medicamentos: processo formativo nos assentamentos do município de Pontão - RS" foi institucionalizado por processo formal e contou com o subsídio do Programa de Iniciação em atividades de Extensão da Universidade Federal da Fronteira Sul - UFFS. As atividades foram executadas durante o primeiro e segundo semestres de 2015.

Todos os recursos humanos envolvidos no projeto foram responsáveis por organizar as temáticas e referências bibliográficas, definir a dinâmica do grupo e auxiliar na construção coletiva dos saberes. As temáticas foram conduzidas pelos acadêmicos, auxiliares e docentes orientadores contando também com a participação da Secretaria de Saúde do Município de Pontão - RS através da enfermeira da unidade básica de saúde, que participou ativamente da viabilização e condução das atividades.

Os temas abordados nos encontros foram distribuídos da seguinte forma:

$1^{\circ}$ encontro: Apresentação do Curso e Automedicação - neste primeiro encontro foi feita uma apresentação do funcionamento do processo formativo-reflexivo, aproveitando esse momento para criação de um vínculo com os participantes e também a realização de uma primeira abordagem sobre a au- 
tomedicação com os presentes.

$2^{\circ}$ encontro: Interações Medicamentosas - no segundo encontro a temática acerca das interações medicamentosas foi amplamente discutida, buscando trazer dos participantes exemplos de hábitos relacionados ao uso dos medicamentos que poderiam oferecer potencial de interações, e desta forma ressaltar a importância de seguir as orientações médicas e farmacêuticas corretamente para evitar essas ocorrências.

$3^{\circ}$ encontro: Uso racional de Antidepressivos - no terceiro encontro, como a temática foi o uso dos antidepressivos, buscou-se estabelecer um caráter de naturalidade na abordagem do tema da depressão, usando para isso algumas estatísticas mundiais, nacionais e regionais, e desta forma a participação dos presentes foi muito proveitosa.

$4^{\circ}$ encontro: Uso racional de Anti-hipertensivos - nesse encontro buscou-se fazer um levantamento prévio voluntário entre os presentes quanto à realização regular de atividades físicas e de hábitos alimentares antes de entrar na temática da hipertensão; a partir das respostas obtidas, foi sendo construída uma linha norteadora de discussão dos diferentes medicamentos dessa classe farmacológica.

$5^{\circ}$ encontro: Uso racional de Anti-inflamatórios não-esteroidais - nesse encontro na discussão estabelecida abordou-se além do uso dos anti-inflamatórios não-esteroidais, um reforço quanto às dúvidas sobre os aspectos de interações e efeitos adversos desses medicamentos, ressaltando os perigos da automedicação e a importância do acompanhamento médico.

$6^{\circ}$ encontro: Plantas Medicinais e Encerramento do Curso - nesse momento final, foram selecionadas algumas plantas nativas para norteamento da discussão, entretanto o diálogo mostrou-se o mais fluido dentre os encontros porque a participação dos presentes foi bastante intensa, com cada um trazendo saberes familiares sobre plantas e usos diferentes, bem como dúvidas sobre validade científica e riscos.

Durante o desenvolvimento dos encontros do projeto evidenciou-se a necessidade de ajuste na dinâmica e na linguagem utilizada, usando-se algumas vezes para uma abordagem linguística mais informal de acordo com o público que estava presente, pois este se alternava entre idosos e adolescentes. A participação mais efetiva foi a do sexo feminino, por serem as mulheres as responsáveis pelos afazeres domésticos e, por possuir essa característica laboral, tinham maior disponibilidade para participar dos encontros.

Exemplificando alguns dos encontros, podemos salientar o terceiro encontro, cujo tema principal era o uso racional de antidepressivos, onde foi possível realizar uma atividade com a participação intensa do público presente, esses receberam a orientação de escrever ou falar sobre o que acreditavam serem momentos de alegria e momentos de tristeza. Com uma plateia muito diversa, foi possível não só 
conhecer os diferentes pontos de vista daquela comunidade, como também a forma com que lidavam com a situação, e quando, efetivamente, buscavam auxílio médico e farmacológico para a situação.

Nessa exemplificação é que é possível concluir a viabilidade da metodologia do processo formativo-reflexivo de Freire à construção de conhecimentos de forma libertária e autônoma. O tema abriu o leque para o debate intenso e a manifestação dos participantes, os quais promoveram juntos a construção do conhecimento referente à alegria e à tristeza. Tal situação também foi a visualização prática de uma formação na qual não é desprezado o conhecimento, o entorno e o dia a dia da comunidade em si, incentivando o debate, a crítica e a construção conjunta dos saberes, os quais depois poderão ser disseminados.

Para o mediador do projeto, tal processo também é de suma importância. Quando colocado na posição de disseminador do conhecimento, no sentido da utilização racional dos medicamentos, também executa um aprendizado e realiza processo de reflexão sobre aquilo que absorve dos participantes. Assim,

"[...] a prática de ensinar que envolve necessariamente a de aprender a de ensinar. A de pensar a própria prática, isto é, a de, tomando distância dela, dela se 'aproximar' para compreendêla melhor. Em última análise, a prática teórica de refletir sobre as relações contraditórias entre prática e teoria" (FREIRE, 2001, p. 205).

Assim, tendo em vista a experiência relatada, da forma como foi conduzida e diante dos resultados dos encontros, pode-se inferir que o processo formativo-reflexivo utilizado para a prática se mostra como uma sistemática extremamente adequada, motivacional e instigadora para os condutores do projeto e libertária, conscientizadora e disseminatória para os participantes (comunidade). Isso porque para os participantes, talvez os mais beneficiados pelo projeto, a forma de condução dos trabalhos permite a conjugação de saberes e experiências que estão além dos limites do conhecimento científico, o que instiga o debate da aplicação da mesma em outros meios e âmbitos e conjuga saberes, formas e modos de interação com os indivíduos. Para a comunidade, há uma latente conscientização sobre o uso de medicamentos, com o implemento de conhecimentos e o reconhecimento de seus saberes populares, os quais serão, certamente disseminados.

Já a libertação será no sentido justamente do reconhecimento dos saberes locais e a liberdade do contato com os condutores do projeto, inclusive na possibilidade de buscarem, a partir de agora, mais informações sobre os assuntos e, no limite, de forma autônoma. Ou seja, nesse processo todos foram beneficiados, numa total conjugação da saberes e competências, mediante um processo formativo-reflexivo o qual poderá, inclusive, ser levado para outras dinâmicas da comunidade e em outros processos nos quais os condutores do projeto poderão participar.

\section{CONSIDERAÇÕES FINAIS}


Portanto, o projeto de uso racional de medicamentos permitiu indubitavelmente a troca de conhecimentos e, principalmente, o esclarecimento da comunidade a respeito do uso de medicamentos. Além disso, as atividades no assentamento Annoni no município de Pontão-RS, contribuíram para o processo de formação acadêmica, no qual foi possível consolidar não só os conhecimentos adquiridos em salas de aula como também maximizar o senso crítico, pois possibilitou aos acadêmicos reconhecerem as diferenças sociais, culturais e econômicas da região.

Outro ponto importante a ser considerado é que essas atividades possam ser ampliadas e implementadas em outras regiões de abrangência da universidade.

\section{REFERÊNCIAS}

[1] Aquino, D.s. Por que o uso racional de medicamentos deve ser uma prioridade? Departamento de Farmácia, Faculdades Integradas da Vitória de Santo Antão, 2007.

[2] Barros, J.a.c. Propaganda de medicamentos: atentado à saúde? São Paulo: Hucitec/Sobravime; 1995.

[3] Bodstein, A.; Lima, V. V. A. De; And Barros, A. M. A. De. A vulnerabilidade do idoso em situações de desastres: necessidade de uma política de resiliência eficaz. Ambiente e sociedade. [online]. 2014, vol.17, n.2 [cited 2016-06-17],pp.157-174. Available from: <http://www.scielo.br/scielo.php?script=sci_arttext\&pid=S1414753X2014000200011\&lng=en\&nrm=iso>. ISSN 1809-4422. http://dx.doi.org/10.1590/S1414-753X2014000200011.

[4] http://bvsms.saude.gov.br/bvs/publicacoes/servicos_farmaceuticos_atencao_basica_saude.pdf - Acesso em 17/60/2016 ás 19:05h.

[5] Brasil. Portaria no 3916/MS/GM, 30 de outubro de 1998. Aprova a Política Nacional de Medicamentos. Brasília, DF: Diário Oficial da República Federativa do Brasil, de 10 de novembro de 1998.

[6] Ministério da Saúde. Envelhecimento e saúde da pessoa idosa. Brasília: Ministério da Saúde.

Cadernos de Atenção Básica, n. 19. Série A. Normas e Manuais Técnicos. 2006.

[7] __-_-_. Prêmio Nacional de Incentivo ao Uso Racional de Medicamentos. Página de Divulgação. Ministério da Saúde. Sistema Único de Saúde. Disponível em:<http://bvsms.saude.gov.br/bvs/premio_medica/oque.php>. Acesso em 07 jun. 2016

[8] Chaves, G.c.; Emmerick, I.; De Pouvourville, N.; De Saint-Denis, T.; Fonseca, A.s.a.; Luiza, V. L. Indicadores de uso racional de medicamentos e acesso a medicamentos: um estudo de caso. Rev. Bras. Farm. 86(3): 97-103, 2005.

[9] Freire, P. Educação em mudança. Rio de Janeiro: Paz e Terra, 1979.

[10] __-_-_. Conscientização: teoria e prática da libertação: uma introdução ao pensamento de Paulo Freire. 3 ed. São Paulo: Moraes, 1980.

[11] ___-_. Pedagogia do oprimido. 17 ed. Rio de Janeiro: Paz Terra, 1999.

[12] _-_-_. Pedagogia dos sonhos possíveis. São Paulo: Editora UNESP, 2001.

[13] _-_-_. Pedagogia da autonomia - saberes necessários à prática educativa. São Paulo: Paz e Terra, 2011.

[14] Gadotti, M. Perspectivas atuais da educação. Porto Alegre: ARTMED, 2000. p. 09-15.

[15] Leite, S. N.; Vieira, M.; Veber, A. P. Estudos de utilização de medicamentos: uma síntese de artigos publicados no Brasil e América Latina. Ciênc. saúde coletiva, Rio de Janeiro, v. 13, supl. p. 793-802, abr. 2008. Disponível em <http://www.scielosp.org/scielo.php?script=sci_arttext\&pid=S1413-81232008000700029\&lng=pt\& nrm=iso>.Acesso em 06 jun 2016.

[16] MSH (Management Science for Health). Managing for Rational Drug Use. In: Managing Drug Supply. The 
REVISTA GUARÁ - Dezembro 2018. ANO VI - NX

Selection, Procurement, Distribution and Use of Pharmaceuticals, PP. 421-429, Connecticut: kumarian Press, 1997.

[17] Oenning, D.; Oliveira, B. V. De; Blatt, C. R. Conhecimento dos pacientes sobre os medicamentos prescritos após consulta médica e dispensação. Ciênc. saúde coletiva, Rio de Janeiro, v. 16, n. 7, p. 3277-3283, jul. 2011. Disponivel em <http://www.scielosp.org/scielo.php?script=sci_arttext\&pid=S1413-81232011000800027\&ln $\mathrm{g}=$ pt\&nrm=iso>. Acesso em 06 jun. 2016.

[18] Wannmacher, L. Condutas baseadas em evidências sobre medicamentos utilizados em atenção primária à saúde. In: Uso Racional de Medicamentos - temas selecionados - série A. normas e manuais técnicos. Ministério da saúde, 2012. 


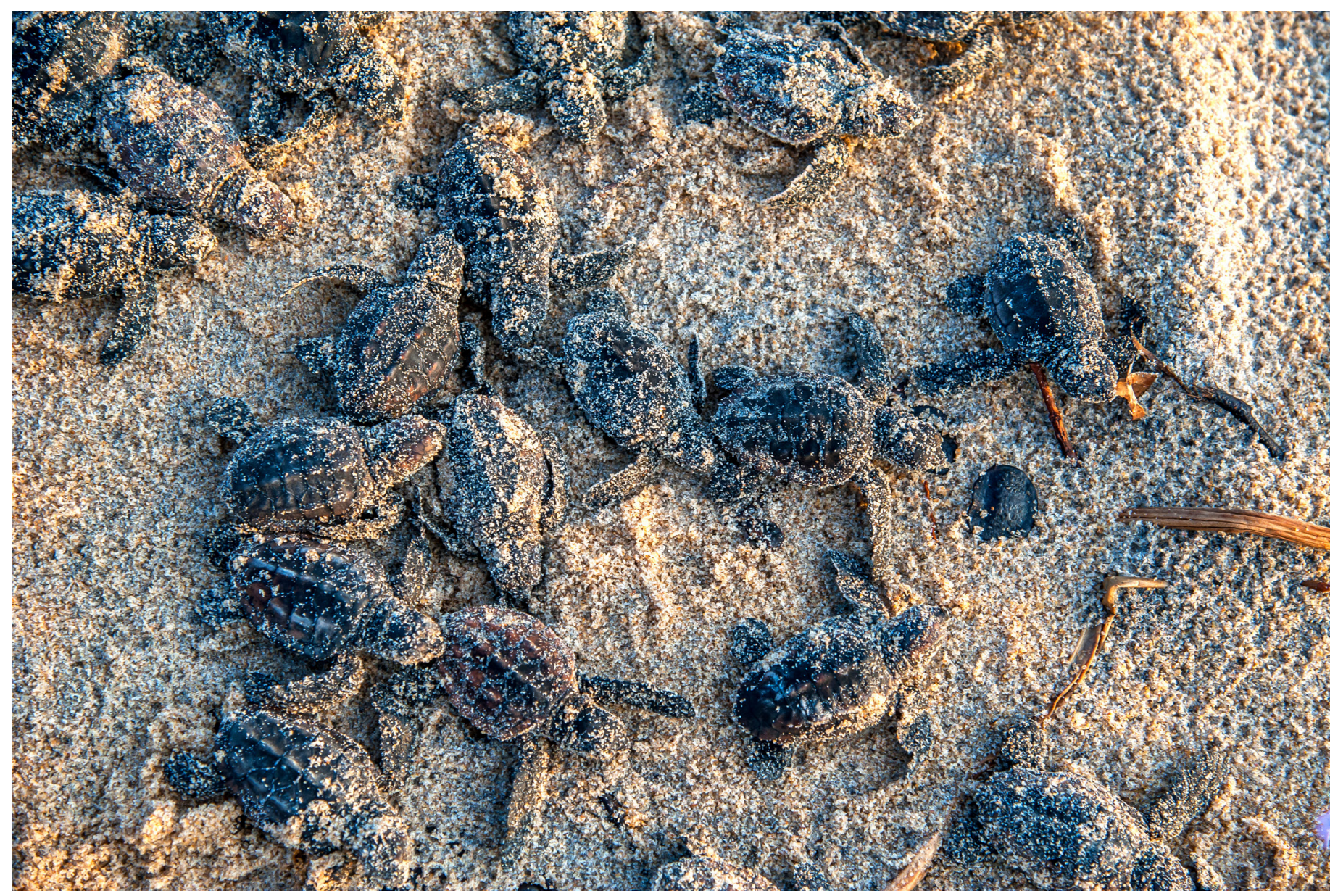

